\title{
Graduates welcome on-road: A culture shift in ambulance preceptorship made clear through retrospective analysis
}

\author{
A. Lazarsfeld-Jensen, D. Bridges \& $H$. Carver
}

\begin{abstract}
A cultural shift in ambulance services has improved the experience of universityeducated paramedics going on-road for the first time in New South Wales. In the "bad old days", graduate paramedics reported routine rites of initiation, including barbed humour and contempt for skills gained in a university setting. Those educated in the on-road vocational system believed universities did not provide a tough enough environment for future paramedics. In this study, data were drawn from two projects involving university-educated paramedics who graduated between 1996 and 2011 and whose novice on-road experiences were several years apart. Comparative retrospective analysis of data provided evidence of a change in attitude towards New South Wales graduates, with an increase in support and inclusion. Novices with greater confidence in their own capacity was evidence of an attitudinal shift. Engaged on-road preceptors who won the respect of the novice was evidence of an increase in support. Inclusion had been a painful issue for both early cohorts of graduates and industry. Failure to fit is associated with attrition. Early in their on-road practice, recent novices reported a sense of belonging. The authors suggest that the shift in attitude could be attributed, first, to a critical mass of graduates on-road, including increasing numbers with postgraduate qualifications, and secondly, the stated preference of ambulance services in Australia to employ graduates.
\end{abstract}

Keywords: paramedic, preceptors, culture, road ready, clinical education.

\section{Background}

Professions in transition do not always welcome newcomers because they are seen as constituting a threat to job security, status and power (Light, 1994). Cultural resistance to novices is found in many uniformed and para-military organisations, where there is a perceived need to maintain rank, boundaries and standards (Archer, 1999). Paramedics

\section{Charles Sturt University}

\section{Correspondence:}

Dr Ann Lazarsfeld-Jensen

Charles Sturt University

N8 School of BMS, Research Station Drive, Bathurst 2795, Australia

Email: alazarsfeld-jensen@csu.edu.au 
currently face the problems of a complex and unresolved identity, and of rapidly changing standards for entry, promotion and job security. Straddling the worlds of healthcare and emergency services, even paramedic uniforms across different services offer a visual variability from vice-admiral to scrubs. In recent research, paramedics characterised themselves and their priorities differently across state borders (Willis, O’Meara, Lazarsfeld-Jensen, \& McCarthy, 2009).

Although paramedics have still not won a good seat at the table of health services professionalism, national registration, which will provide better consistency and governance, is not far off. However, registration alone will not bring cohesion to a fragmented quasi-profession. An attempt to resolve the issue of portability of qualifications between services in different states, for example, may encounter cultural resistance rather than legal obstacles. Positive cultural change, in terms of inclusion and equity, is essential to ease professional movement between states as well as address problems of attrition and stress. Paramedicine is seen as a "sexy" profession, often dramatised by the media, and it has an exciting future as it diversifies to meet social needs. However, anecdotal evidence that students and new graduates felt anxiety about facing what they perceived as a hostile culture precipitated research into this phenomenon.

It has been argued by academics that on-road preceptorship was essential to complete the graduate's education, and a university degree did not make graduates "road ready" (Willis et al., 2009). Research showed that some graduates and their preceptors saw on-road training as a faster, cheaper and more credible option than a full-time degree program followed by an on-road preceptorship.

Paramedicine is emerging from some of the same teething problems as those experienced by other professions that have adopted a higher education entry standard. These issues include medical dominance (Germov, 2009), the lack of academic leaders with both practice currency and credibility in academia (Kerry, Pontin, \& Moore, 2004) and industrial ambivalence due to the resistance of vocationally trained veterans who understandably do not want late-career training demands made on their time and finances (Andrew, Ferguson, Wilkie, Corcoran, \& Simpson, 2009).

Students' choices to identify with on-road training, and often to hide their graduate identity, is not surprising in light of evidence that their university lecturers may not have resolved their own career identity issues (Andrews et al., 2009). Andrews et al. (2009) showed that practice currency was regarded as more personally significant, and the loss of practice was often mourned by new academics who were reluctant to embrace an academic agenda. They suggested that in some new professions, there was a danger of perpetuating vocational models instead of developing the depth of discourse expected in higher education. Defining the identity and the theoretical discourse of paramedicine has hardly begun in this new academic discipline (Willis et al., 2009). However, the national educational research work of Willis et al. (2009) did identify on-road preceptorship as the signature pedagogy of paramedics (Shulman, 2005b) and the primary point of connection between theory and practice for graduates. As such, 
the facilitation of good on-road preceptorship is critical to the future of graduates and ensures that the knowledge of those paramedics who entered by vocational pathways is valued and utilised.

It is a continuing challenge to educators and ambulance services to provide clinical placements of adequate duration with supervision for paramedic students, particularly in cases such as Charles Sturt University (CSU), with 800 enrolments. In 2007, problems in translating theory into practice were flagged by research in Victoria where the dearth of clinical placements demonstrated poor matching of learning objectives to case exposure (Michau, Roberts, Williams, \& Boyle, 2009). Despite limited learning opportunities, students expressed satisfaction with their placement. In a similar study at Monash University, it was found that many placements were not seen as positive, either clinically or educationally (Boyle, Williams, Cooper, Adams, \& Alford, 2008). This second study focused on gender inclusion problems by exploring the physical size of female undergraduates whose capacity had been questioned on-road. Other problems included some gate-keeping behaviour by clinical preceptors, which restricted learning opportunities. Students reported they were not made welcome, they were treated with disdain, there were sexist attitudes, students were excluded from hands-on experience and clinical instructors were not consistently available. Students' willingness to endure such indignities was confirmed in subsequent projects reported in this paper.

The Monash findings matched anecdotal evidence from students' clinical placement journals at CSU $(n=100)$. However, it was also observed that generations of students at CSU perpetuated stories of on-road hazing, particularly in the clinical placement lectures where cohorts from first to third year were merged. It was also observed in focus groups for unrelated research that paramedic preceptors repeated anecdotes about students. In 2010, the practice of merging cohorts ceased, and there seemed to be a shift in the experiences that students reported in their reflective journals. This prompted a review of extant data.

\section{Methods}

Two discrete qualitative inductive research projects were reviewed in this retrospective analysis of cohorts of higher education paramedics. The first project conducted, "Transitions: Command culture and autonomous paramedic practice" (LazarsfeldJensen, Bridges, \& Loftus, 2011), looked at the transition to work in the protocol-driven work culture of paramedics and the adaptation experienced by graduates educated for autonomous practice. The second project, "Fasttrack: Evidence-based strategies for optimising short-term paramedic internships", was an examination of the on-road experience of students on clinical placement. Both projects were conducted by research teams at CSU, in order to search out best practice in clinical placement education.

Transitions was a qualitative ethnographic study where semi-structured telephone interviews were conducted with CSU paramedic graduates working in the NSW Ambulance Service $(n=8)$. Most interviewees had been in the service only a short time (average, 2.5 years), although it was considered informative to include older graduates, 
including one who had worked as a vocationally educated paramedic before joining the first cohort of distance-education students at CSU in 1995. Graduates in the study had received degrees between the years of 1996 and 2008.

The Fasttrack research looked at more than 100 student placement journals that were very similar in content but selected only the most descriptive examples $(n=13)$ of those that had been maintained progressively by students in the final semester of their third year, following their second clinical placement. These de-identified samples were a rich record of the students' clinical placements. The selected journals were analysed thematically. Further information was elicited in personal inductive interviews $(n=3)$. Students in this study were ready for recruitment in 2011. Saturation of themes in the two discrete studies was reached quickly. The students' reflections in their journals and during interviews strongly emphasised paramedic culture.

Both projects were approved by Charles Sturt University's Human Research Ethics Committees (406/2010/16, 2010/018, respectively).

\section{Findings and discussion}

The graduates interviewed in Transitions spoke openly about the challenges they faced during their probationary period. As well as the challenges of marrying clinical skills with the need for expert interpersonal communication skills and the fast-paced nature of the work, graduates also experienced NSW Ambulance Service colleagues as having higher expectations of them than they did of paramedics from a vocational entry, yet paradoxically underestimating graduates' capacity. The on-road culture of "do it my way" conflicted with the university education graduates had received and made the new probationary officers uncomfortable.

Among paramedic students, even where there was no suggestion of deliberate exclusion, there were reports of limited opportunities to refine their clinical skills during placements (Boyle et al., 2008; Lazarsfeld-Jensen, 2010; Lazarsfeld-Jensen et al., 2011; Michau et al., 2009; Waxman \& Williams, 2006). At times, as a coping strategy, graduate recruits hid their university background from colleagues and almost colluded with their oppressors in developing antipathy for university preparation along with their own explanations for their preference for on-road training.

The main issue for graduates — and until recently CSU was the main source of graduate paramedics in NSW—-was the resentment provoked by their accelerated promotion, which they believed led to poor treatment. After induction into the ambulance service, graduates are able to cannulate and administer medications, and they qualify as paramedics two years ahead of vocationally-trained colleagues. This arrangement puts them in a senior position, despite the traditional priority given to length of service. This tension was clearly identified by CSU graduates during their early years in the NSW Ambulance Service: 
I have more issues now than ... I did as a probationer, with people having issues against CSU students ... because I'm now qualified, especially from people who had been in a job for longer than I am, but I am more senior than them, so there are big issues against that. I am currently having big problems with my current partner who likes to tell me that I am still a probationer in her eyes. (Lesley)

The resistance to graduate education extended beyond accelerated career pathways to a general attitude that university education was unnecessary and even counterproductive. Six out of the eight research participants felt that there was significant resistance to CSU graduates, with older officers openly dismissive of the Bachelor of Clinical Practice (Paramedic) degree. The participants reported a fear of discrimination, which they believed resulted from a stigma attached to the CSU degree program. Some graduates, once recruited, actively concealed their university education and qualifications from their colleagues.

Stigma against CSU students was said to impact on the clinical placement learning experience, with one graduate saying that she had been denied the opportunity to learn whilst on clinical placement:

When I did my placement the ... two paramedics pretty much ignored me, and I didn't do anything because they didn't want to know me, so I just sat in the back of the ambulance and took a blood pressure or two. (Ashley)

One graduate reported a lack of support that was dangerous to patients and the probationer: Well, we did a job once ... I was pretty much brand new, fresh. And I was working with a guy who didn't really want to train me or help me out at all, and we went to a person who was like about to die. He was super, super sick and had heaps of stuff going on with him ... I just didn't know where to start because he had so many things going on. He couldn't breathe, he had chest pain, couldn't walk, he just was super, super sick, and I kind of looked at him for a bit of guidance, like okay where do I start? ... and he just looked at me and just didn't give me anything ... I just froze.... [Later, another officer] sat down and went over the job, and she told me what it was and what my priorities were, like she helped me out instead of my training officer... I kind of struggled for a few weeks with him. (Alex)

Many graduates said they were afraid of prejudice, actively worked to avoid being identified as a graduate and had difficulty finding a balance between assertiveness and submission to other paramedics. Respondents reported verbal reproaches that clearly expressed that the CSU degree "doesn't mean anything":

I was told that at first: "You're too young", "The degree doesn't mean anything", "You should be out there doing other stuff", but I just kept on pushing on because I knew [what] I wanted. (Ashley)

The change in professional expectations of ambulance services in Australia was flagged as a reason why some "old-timers" were resistant to CSU graduates. For those without a degree, the rising educational expectations were thought to be threatening: 
A lot of people on the job, to be fair, don't have a degree, so perhaps somebody who comes in who does have a degree can be confronting for them and can also be intimidating for them. They have the confidence on the road... [but] they haven't got a university based knowledge ... so whether that makes it more of a challenge for them, I don't know. (Pat) I faced a lot of resistance to the idea of tertiary education sort of in this field of work. ... I think some people saw me as a threat. (Taylor)

One graduate suggested the "macho" culture of the ambulance service fuelled opposition to CSU graduates:

Ifyou have the degree they're like, "Well, just because you've got a degree doesn't mean you know what I've been through", and it's a macho sort of ego thing that you have to deal with sometimes, and that makes it hard. (Ashley)

This graduate went on to discuss the issue of ambulance paramedics not knowing what a CSU graduate was authorised to do in the course of their work. The unfamiliarity about what the graduate is permitted to do, or not do, was causing confusion. Other probationers, not from CSU, have other authorisation standards:

There didn't seem to be any education [shared understanding between] the other officers [about] what I was allowed to do, which led to tense situations on ... certain jobs where people had just assumed I was a normal trainee, and they saw me as operating outside of my authorised protocols. (Taylor)

Some graduates interpreted the resistance of colleagues as the hazing that occurs in many uniformed services as a rite of passage:

Until you have been at a station for a while, and they get to know you, and they see that you do know your stuff, and you earn your kind of stripes ... they treat you different. And I changed stations about three weeks ago, so I am just starting to go through that again. (Lesley)

Whether it is labelled hazing or bullying, it is a strategy that is now rejected socially. Social objection to hazing in uniformed service organisations has been reinforced by media reporting, such as the NSW parliamentary questions raised in relation to the suicide of Christine Hodder at Cowra in 2005, where there were widespread allegations of gender-based bullying, and the 2011 Report into the Review of the Treatment of Women in the Australian Defence Force, which was particularly concerned with the safe passage of cadets (Human Rights Commission, 2011).

Hazing did not occur where preceptors embraced an educative role. In both reflective journals and qualitative interviews, students in the Fasttrack research identified their on-road educators as supportive mentors. There was a learning hierarchy regardless of the preceptor's training, and where the relationship was free of defensiveness, students rated the opportunity to participate actively in on-road events to be integral to their learning experience and important to the consolidation of their skills and knowledge. 
Clinical placement was understood by students as necessary to prepare them for their careers as professional practitioners, to build confidence and to provide personal motivation. The role of the paramedic crew that the student was placed with, and most significantly the relationship with the preceptor, was identified by students as fundamental to the success of their placement. Clearly, Transitions research participants emphasised defensive and aggressive attitudes, while Fasttrack research participants' experiences were more positive.

The reports of Fasttrack participants reinforced the concept that experiential (on-road) learning is the signature pedagogy of paramedic practice (Shulman, 1986, 1998, 2004, 2005a, 2005b), and the ultimate success and retention of a graduate in the industry depended on the quality of on-road preceptorship and their adaptation to the culture of the ambulance service. Students' journal reflections also focused on the relational rather than the clinical education aspects of their on-road clinical placement.

In the later (Fasttrack) study, the anecdotal legacy of stigmatisation was still present during student placements. Although none of the participants experienced rejection, they feared it:

I am fully aware of an attitude out in the profession that still holds a certain grudge against young trainees that have pursued a university degree. My partner experienced such a difficult situation ... which caused an amount of distress ... if I meet a paramedic that is closed and non-accommodating to the point of being rude, then I know I will feel unwelcome and as a result not take full advantage of my internship. (Respondent 01)

Talking with some of the paramedics, it appears that CSU students tend to be frowned upon for reasons such as being "know-it-alls" and being clinically knowledgeable with no people skills. I didn't feel any bad feelings towards myself. (Respondent 03)

However, the students overwhelmingly experienced acceptance and support. The student below was given support by the whole paramedic team after two traumatic jobs that ended in the deaths of patients. The care taken by the paramedics was professional and helped to consolidate the student's learning as well as offset a potential trauma:

Over the last four days, I've seen a lot of things I didn't expect to see ... a patient in respiratory arrest post hanging, and an MVA [motor vehicle accident] car vs. truck ... Due to the nature of the events, my clinical mentors, ICPs [intensive care paramedics] and station managers have all pulled me aside individually and given me an opportunity to express how I felt after seeing everything that I saw. Not only did they ask me, both my station manager and my clinical mentors gave my number to an independent counselling service so I was able to talk to someone who wasn't there and wasn't affected by the job in any way... I was also really comforted by the support that was shown by my clinical mentors not only on the day but even in the weeks post the event while I was on placement. However, they still check in with me every so often via phone calls or texts messages to see how I'm going and if I'm coping, which I'm happy to say I'm dealing with things very well. (Respondent 11) 
The modelling of team work and the inclusivity reported in Fasttrack contrasts with the isolation and rejection experienced by graduates in the Transitions study. Teams that were working well together were seen by students as being easily able to integrate the student learner:

The paramedics all work closely together, and there is this great team work mentality, and I think this is a big part of creating a healthy workplace ... I was able to witness a debrief between two separate ambulance crews; I was at Westmead hospital when we heard that a code two was coming in [who eventually died]. After this, both of the crews debriefed about the event and getting to watch this was incredible, as it showed me that the paramedics really worked well together. (Respondent 06)

We have had the same two partners on alternate days. This has been great to build up rapport and also establish myself and my skills to gain their trust. Working as a part of a team has been one the best things from this prac. (Respondent 07)

This year's practicum I felt more like I was part of the team, rather than just an observer. The paramedics got me involved in carrying out things such as radio checks, sign on, vehicle checks, and I felt more like a probationer than a student. (Respondent 10)

I was able to develop a great respect for both of them [preceptor and team] as well as develop really good friendships with both of them. As a result, I really enjoyed being part of their team. (Respondent 11)

Students were able to maximise their learning by participating as a team member, by making a useful contribution and by observing the team work together effectively. In this way, the team provided an excellent learning environment for the student:

On the whole, most paramedics have been more than happy to include me as part of the team, allowing me to practise skills, ask questions and even test me ... These paramedics are also more than willing to answer any question ... as a team was great in knowing that you had your partner to cover your back, or bounce things off. (Respondent 12)

The student below learnt how to work as an effective member of a paramedic team and also learnt to work with allied professionals:

Throughout my clinical placement I have noticed that team work is an enormous part of ambulance culture. Not only are you constantly working with your partner, constantly asking advice and aid from them, but you are also quite often working with other ambulance crews. During this prac so far, I have found myself working with other crews from Blacktown station, as well as other station [sic] close by. We have also worked alongside police and fire fighters. (Respondent 09)

Students interviewed for the Fasttrack study said that they were welcomed into a positive and supportive learning environment:

I was surprised how they eased me into it. I was prepared to be just thrown in to the deep end, maybe being stuck with someone that didn't want to teach me; [however], the first day, they took like about four hours to go through all their oxy vivas, and they even put 
me through a scenario ... and gave me some tips, went through the truck and showed me where everything was ... they were excellent ... and I thought that was good of them. Like, they were all really good to me. (Interviewee 01).

I was really lucky because I went to a station that ... didn't leave me behind on jobs ... got me involved as I became more confident. (Interviewee 02)

The intensive care paramedic was very generous and asked a lot about what we had learned so far, and confirmed why we were being taught the way we are ... and he very much encouraged thinking. (Interviewee 03)

Students said that their learning experience on placement was so positive that it increased confidence and motivation. It allowed them to see that the role was what they wanted in a career:

Before placement I was ... losing my confidence. Like, do I really want to do this, am I made out for it? And but yeah, I enjoy prac a lot, and it made me think that I can do it ... like prac increased my motivation too, like before prac, I was so drained and like sick of figures, text books and but since I went on prac, I was much more motivated to go do it, if that makes sense? (Interviewee 01)

\section{Conclusion}

This research revealed that effective and dedicated preceptors are essential to the success of graduates. Although ambulance service policy and practice in regards to supporting graduates has continued to evolve, the success of placements and the integration of graduates still depends on individual preceptors. Students function best with those paramedics who take a leading educative role, who are informed about tertiary education, who take into account novice strengths and limitations, and who are both tolerant and supportive of the generational nuances. While the more positive reception reported by recent graduates augurs well for the future, the need for dedicated preceptors in a role that is both respected and rewarded is the key to seamless inclusion.

In this analysis, it was clear that university-educated paramedics are no longer the exception. New graduates are now more likely to be supervised on-road by another graduate, or by someone undertaking postgraduate qualifications. Graduates have confidence in their knowledge of disease processes as well as a broad base in anatomy and physiology, which is respected and utilised by new colleagues. Their ability to research disease and clinical interventions is also regarded as a bonus by colleagues.

Although it could be argued that this analysis had a negative bias, and the samples were small over two discrete projects, it is also significant that the research was prompted by persistently negative student anecdotes of early on-road experiences. In both the research projects reported here, the role of good preceptors was the common theme. Since the completion of this research, CSU has conducted some training for preceptors, and the NSW Ambulance Service is developing its own educational program and policies. The relationship between the university and the ambulance service has been strengthened 
by a co-appointment of a workplace learning facilitator to improve student experiences in NSW. A collaborative approach to policy and research at this significant interface will ensure that graduates and their preceptors are guided in best practice.

Paramedicine is a profession that is likely to continue to evolve to meet the needs of Australia's remote and aging population. Graduates will be in the frontline of unique health roles and occupations 20 years hence. Retention of highly-trained workers remains a challenge for employers, and the usual answer of reducing stress is not necessarily the key. While shared trauma causes stress, it also builds those familiar bonds of mateship that sustain individuals in rescue and protection roles. Belonging is central to so many professionals' work satisfaction. A key to sustainability is cultural transformation to provide greater inclusion and support for newcomers. For graduates, the challenge is to make a strong social and emotional connection to the job to justify their expensive education - a goal they share with heavily-invested educators and employers.

\section{References}

Andrew, N., Ferguson, D., Wilkie, G., Corcoran, T., \& Simpson, L. (2009).

Developing professional identity in nursing academics: The role of communities of practice. Nurse Education Today, 29(6), 607-611.

Archer, D. (1999). Exploring bullying culture in the para-military organisation. International Journal of Manpower, 20(1/2), 94-105.

Boyle, M., Williams, B., Cooper, J., Adams, B., \& Alford, K. (2008). Ambulance clinical placements: A pilot study of students' experience. BMC Online, 8(19). Retrieved from http://www.pubmedcentral.nih.gov/articlerender. fcgi?artid $=2330039$

Germov, J. (2009). Challenges to medical dominance. In J. Germov

(Ed.), Second opinion: An introduction to health sociology (pp. 392-415).

Melbourne, Australia: Oxford University Press.

Human Rights Commission. (2011). Review into the treatment of women in the Australian Defence Force Academy. Retrieved from http://humanrights.gov.au/ defencereview/ADFA_report/ADFA_2011.pdf

Kenny, G., Pontin, D., \& Moore, L. (2004). Negotiating socialisation: The journey of novice nurse academics into higher education. Nurse Education Today, 24(8), 629-637.

Lazarsfeld-Jensen, A. (2010). Starting young: The challenge of developing graduates' road readiness. Journal of Paramedic Practice, 2(8), 270-274.

Lazarsfeld-Jensen, A., Bridges, D. R., \& Loftus, S. (2011). Transitions: Command culture and autonomous paramedic practice. Bathurst, Australia: RIPPLE, Charles Sturt University. Retrieved from http://csusap.csu.edu.au/ cmcewen/Documents/ TransitionsReport.pdf 


\section{A CULTURE SHIFT IN AMBULANCE PRECEPTORSHIP}

Light, D. (1994). A framework for professions in transition. In T. Johnson, G. Larkin, \& M. Saks (Eds.), Health professions and the state in Europe (pp. 25-41). London: Routledge.

Michau, R., Roberts, S., Williams, B., \& Boyle, M. (2009). An investigation of theory-practice gap in undergraduate paramedic education. BMC Medical Education, 9(1), 23. doi:10.1186/1472-6920-9-23

Shulman, L. S. (1986). Those who understand: Knowledge growth in teaching. Educational Researcher, 15(2), 4.

Shulman, L. S. (1998). Theory, practice, and the education of professionals. Elementary School Journal, 98(5), 511.

Shulman, L. S. (2004). The wisdom of practice: Essays on teaching, learning, and learning to teach. San Francisco: Jossey-Bass.

Shulman, L. S. (2005a). Pedagogies of uncertainty. Liberal Education, 91(Summer), 18-25.

Shulman, L. S. (2005b, February). The signature pedagogies of the professions of law, medicine, engineering, and the clergy: Potential lessons for the education of teachers. Paper presented at the meeting of the National Research Council Center for Education, Irvine, California.

Waxman, A., \& Williams, B. (2006). Paramedic pre-employment education and the concerns of our future: What are our expectations? Journal of Emergency Primary Health Care, 4(4). Retrieved from http://ro.ecu.edu.au/jephc/vol4/iss $4 / 4$

Willis, E., O’Meara, P., Lazarsfeld-Jensen, A., \& McCarthy, C. (2009). Paramedic education: Developing depth through networks and evidence-based research. Retrieved from www.altc.edu.au 